\title{
Conociendo las Unidades de Saúde Familiar: otro modelo de Medicina de Familia
}

\author{
Ricardo Enrique Reolid Martínez a , María Flores Copete ${ }^{a}$, Francisco Jesús Escobar \\ Rabadán ${ }^{b}$, Susana Gomes Corte-Real ${ }^{c}$, Vanda Cristina de Jesus Ramalho Proençac.
}

\author{
a Médico Interno Residente \\ de Medicina de Familia \\ y Comunitaria. Gerencia \\ Área Integral de Albacete. \\ CS Zona 4. Albacete. España. \\ ${ }^{b}$ Médico de Familia y \\ Comunitaria. Gerencia Área \\ Integral de Albacete. CS \\ Zona 4. Albacete. España. \\ c Médico de Familia y \\ Comunitaria. Unidades de \\ Saúde Familiar de São Julião. \\ Oeiras. Portugal.
}

Correspondencia:

Ricardo Enrique Reolid

Martínez.

Centro de Salud Zona IV. C/ Seminario, 4. CP: 02006.

Albacete. España.

Correo electrónico:

ricardoerm@hotmail.com .

Recibido el 8 de mayo de 2015.

Aceptado para su publicación el 2 de junio de 2015.

\begin{abstract}
RESUMEN
Las Unidades de Saudé Familiar (USF) suponen un modelo de Atención Primaria desconocido para muchos. Nacidas dentro de la reforma del sistema nacional sanitario portugués, surgen ante la necesidad de proporcionar unos cuidados de salud de calidad a los ciudadanos, mejorando la satisfacción tanto de los propios usuarios como de los profesionales que trabajan en ella. En este artículo trataremos de explicar el funcionamiento de estas USF gracias a la oportunidad que tuvimos los autores del mismo de compartir consulta en la USF de Sao Juliao-Oeiras.
\end{abstract}

Palabras claves: Atención Primaria de Salud. Medicina Familiar y Comunitaria. España. Portugal.

\section{ABSTRACT}

Knowing the Unidades de Saúde Familiar: another model of family medicine Unidades de Saude Familiar (USF) represent a model of primary care unknown to many people. Born within the reform of the Portuguese national health system, they arise from the need to provide a quality health care to citizens, improving the satisfaction of both users and professionals who work in it. This article will try to explain the working of these USF thanks to the opportunity to share medical consultation that the authors had at the USF-Oeiras Sao Juliao.

Key words: Primary Health Care. Family Practice. Spain. Portugal

\section{INTRODUCCIÓN}

A raíz de la crisis económica de los últimos años, los países se han visto obligados a realizar una serie de reformas, debido a los cambios sociales y económicos surgidos, no siendo la Sanidad ajena a las mismas.

En el Congreso Regional de Atención Primaria del año 2012 celebrado en Albacete, una de las ponencias que mostraba mayor expectación era la presentada por el Doctor Luis Pisco bajo el título "Otros modelos de organización y gestión", dentro de la Mesa de Gestión Sanitaria. En ella se explicaba la reforma de la Atención Primaria vivida en Portugal, donde el pilar fundamental fue la implementación de las Unidades de Saúde Familiar o Unidades de Salud Familiar (en adelante, USF).

Las USF consisten en pequeños equipos multiprofesionales, autogestionados, formados de forma voluntaria por equipos de tres a ocho médicos de familia, acompañados por un mismo número de enfermeras de familia y profesionales administrativos. Abarcan poblaciones de entre 4.000 y 14.000 personas. Disponen de autonomía técnica, funcional y organizativa con un sistema de pago mixto, basado en incentivos financieros y profesionales y, lo 
más importante, sensibles a la productividad, a la accesibilidad y a la calidad. La misión y responsabilidad de las USF es mejorar la atención a la salud de los pacientes basándose en la accesibilidad y la atención continuada.

Con motivo del Congreso mencionado anteriormente, tuvimos la oportunidad de intercambiar ideas y opiniones con el Doctor Luis Pisco. Desde el año 2005, desempeña la labor de Coordinador Nacional de la Unidad para la Reforma de la Atención Primaria en Portugal. Tras volver a coincidir en posteriores congresos, nos brindó la posibilidad de poder visitar estas USF, y conocer de este modo una nueva forma de Atención para la Salud.

\section{HISTORIA DE LAS UNIDADES DE SAÚDE FAMILIAR}

En el año 1993 fue publicado el nuevo Decreto-Lei $n^{\circ}$ 157/99, de 10 de Maio por el cual se aprobó la formación y creación de las unidades integradas de cuidados de salud, destinadas a mejorar la articulación entre los centros de salud y los hospitales ${ }^{1}$.

Posteriormente, en el año 1999 son creados los centros de salud de "terceira geração", compuestos por personas colectivas de derecho público, integradas en el sistema nacional de salud con autonomía técnica, administrativa y financiera bajo la superintendencia y tutela del Ministério da Saúde.

De una revolución organizativa más reciente derivó el Programa del XVII Gobierno Constitucional que reconoció los Cuidados de Saúde Primários (CSP) como el pilar central del Sistema de Salud, y en ese sentido fue creada una missão para os cuidados de saúde primários (resolución del consejo de ministros $\mathrm{N}^{\circ} 157 / 2005$ ).

Finalmente las primeras USF surgen en 2007 , dando cuerpo a la reforma de los CSP bajo el DecretoLei $n^{\circ}$ 298/2007, de 22 de agosto. En 2008 se crean los Agrupamento de Centros de Saúde (ACES) Decreto-Lei $28 / 2008$ de 22 de Febrero, cuyo objetivo es una gestión rigurosa y equilibrada y mejoría en el acceso a los cuidados de salud ${ }^{2}$.

\section{MUNICIPIO DE OEIRAS}

Para un Médico de Familia (MF), el conocimiento de su lista de pacientes y la comunidad en la que se inserta es de primordial importancia, ya que así conseguirá comprender e intervenir, de la forma más adecuada, usando los medios disponibles. De hecho, las características de una población varían de forma relevante con la localización geográfica, así como con algunos localizadores (demográficos, socioculturales y económicos) y recursos de salud. Estos tienen un valor inestimable en el estudio y caracterización de la población objetivo.

\section{Caracterización geográfica}

El municipio de Oeiras tiene un área de 45.850 metros cuadrados y se trata de uno de los $18 \mathrm{mu}-$ nicipios que constituyen el Área Metropolitana de Lisboa (AML), con un 1,6\% de su superficie. Se sitúa en la margen norte del río Tajo, estando delimitado por los municipios de Cascais al oeste, Sintra y Amadora al Norte, Lisboa al Este y al sur por el estuario del Tajo. Actualmente está constituido por 10 freguesias: Alges, Barcarena, Carnaxide, Caxias, Cruz, Quebrada/Dafundo, Linda- a-Velha, Oeiras y São Juliâo da Barra, Paço de Arcos, Porto Salvo y Queixas ${ }^{3}$.

Caracterización demográfica, socioeconómica y cultural

El segundo registro de censo de 2011 de la población residente en el municipio de Oeiras fue de 172.120 habitantes, mostrando una densidad poblacional $\left(3.749,7 \mathrm{hab} / \mathrm{km}^{2}\right)$ superior a la de Portugal $\left(114,5 \mathrm{hab} / \mathrm{km}^{2}\right)$. En la división por géneros se vio que un $53,4 \%$ eran mujeres, mientras que por edad la mayoría de la población se situaba en el rango entre 25 y 64 años $(55,8 \%)$, seguida por igual o mayor de 65 años $(19,2 \%)$ y desde 0 a 14 años (15,4\%).

Entre 2001 y 2011, se verificó un crecimiento demográfico de 6,2 \% en la población residente. El envejecimiento demográfico se ha acentuado en los últimos años: de 57 ancianos por cada 100 jóvenes en 1991 se pasó a 106 en 2001 y a 124 en 2011. Esta realidad puede representar un porcentaje cada vez mayor de población pluripatológica 
y, consecuentemente, más utilizadora de los servicios de salud. Paralelamente, se verificó un aumento del índice de dependencia total de los ancianos, pasando de un 20,9\% en 2001 a un 29,3\% en 2011.

En el Municipio de Oeiras, la población extranjera con estatuto legal de residente disminuyó de 10.461 a 10.100 , entre 2008 y 2011 . Siguiendo los centros de 2011, la mayoría era del continente americano $(37,1 \%)$ y de estos el $95 \%$ eran de Brasil, seguidos del continente africano 34,3\%, europeo 22,6 \%, y por último de Asia 5,9\%.

Los núcleos familiares están constituidos por una media de 2,4 personas, predominando las familias formadas por uno $(27,3 \%)$ o dos miembros $(33,5 \%)$.

Sobre el grado de escolaridad de la población, constaba una tasa de analfabetismo de 2,2 \% (inferior al 5,2 \% nacional) y con un porcentaje de personas que completaron la educación superior del $31,8 \%\left(15,4 \%\right.$ a nivel nacional) ${ }^{4-6}$.

\section{ACES LISBOA OCIDENTAL E OEIRAS}

Los ACES creados en febreros de 2008 son servicios públicos de salud con autonomía administrativa, y constituidos por varias unidades funcionales, que agrupan uno o más centros de salud, y que tienen por misión garantizar los cuidados de salud primarios a una población de una determinada área geográfica.
En nuestro caso particular, la USF São Juliao pertenece a la ACES Lisboa Ocidental y Oeiras. Este ACES engloba las siguientes Unidades Funcionales, cada una con la misión de garantizar una serie de cuidados específicos (figura 1):

- $\quad$ Nueve Unidades de Salud Familiar (USF) y seis Unidades de Salud de Cuidados Personalizados (UCSP). Son unidades para los cuidados de salud a la persona y la familia. Se diferencian entre sí únicamente en el nivel de desarrollo de la dinámica de equipo. Mientras que las primeras están formadas por médicos que trabajan juntos para lograr unos determinados objetivos, en las UCSP los médicos son independientes los unos de los otros. Ambas tienen libertad de designación en función de los recursos disponibles.

- Dos Unidades de Cuidados Continuados (UCC), responsables de los cuidados de la comunidad. Apoyan y complementan a las USF y las UCSP. Su principal objetivo es la defensa y la promoción de la salud colectiva.

- Una Unidad de Salud Pública (USP). Se trata de la unidad de administración de salud que promueven las estrategias locales de salud de ámbito comunitario y de autoridad de salud.

- Una Unidad de Recursos Asistenciales Compartidos (URAP), encargada de dirigir y coordinar los recursos asistenciales de cada ACES, apoyando al resto de las USF.

- Incluye además unidades de apoyo, una unidad de apoyo a la gestión (UAG), que supervisa el funcionamiento de toda la organización para garantizar que existen condiciones materiales y objetivas para que cada unidad cumpla su misión, y un gabinete del ciudadano.

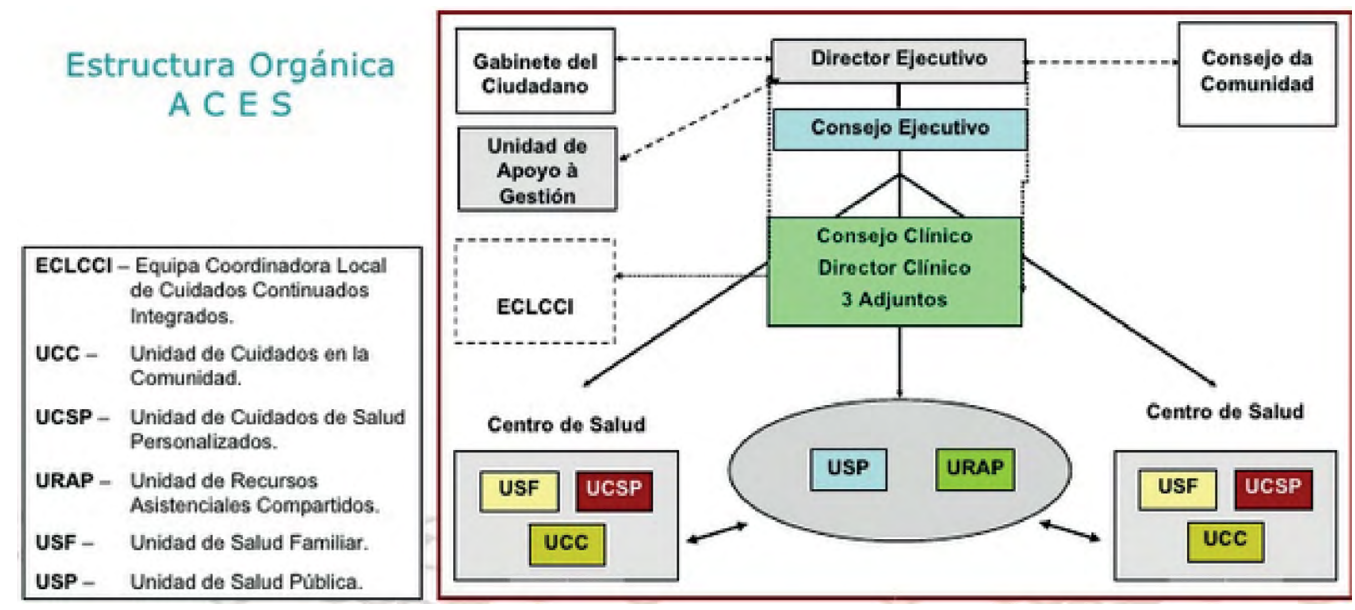

Figura 1. Estructura de los Agrupamento de Centros de Saúde (ACES) 


\section{ESTANCIA EN UNIDAD DE SALUD FUNCIONAL DE OEIRAS}

Durante el mes de julio de 2014, tras su aprobación por la Unidad Docente de la Gerencia de Atención Primaria, tuvimos la oportunidad de realizar una rotación externa en la Unidad de Salud Funcional de Oeiras. Los objetivos marcados en la Rotación Externa, se cumplieron con creces.

En primer lugar conocimos un sistema sanitario totalmente distinto al nuestro. Dentro de la Reforma de Sistema de Atención Primaria en Portugal, una de las medidas realizadas fue la creación de las USF tal y como se mencionó previamente.

Las USF se crean de manera voluntaria por un equipo de profesionales para satisfacer una necesidad de cuidados de salud a una determinada población. El modelo de las USF tiene unas características fijas como son la autonomía técnica, el trabajo multiprofesional y el sistema por incentivos. No obstante, existen diferencias entre ellas en cuanto a los modelos retributivos y de incentivos profesionales y sobre el modelo de financiación y su estatus legal de USF-A, USF-B o USF-C.

- USF-A: Corresponde a la fase de aprendizaje y mejora del trabajo del equipo de salud. Se considera como el primer paso en aquellos equipos donde no existe ninguna tradición o práctica de actuación técnica y científica en la atención a la salud.

- USF-B: Modelo donde los equipos gozan de una mayor madurez organizativa y donde el trabajo en equipo es una práctica efectiva, en la que cada uno de los miembros están dispuestos a aceptar unos objetivos de rendimientos más exigentes.

- USF-C: En este tipo de USF existe un programa de contrato. Los equipos pueden ser del sector público o pertenecer al sector privado, cooperativo o social. Se trata de un modelo experimental con carácter complementario pendiente de ser regulado por ley?

En nuestro caso, se nos asignó la USF de Sao Juliao-Oeiras. Esta fue inaugurada en el año 2007 con el fin de atender a la población residente en las freguesias de São Julião da Barra y de Porto Salvo, correspondientes al Municipio de Oeiras y teniendo como objetivos prestar unos cuidados de salud de calidad a la población inscrita, aumentando el grado de satisfacción de los pacientes y profesionales teniendo como pilares la accesibilidad, la promoción y la prevención de la salud ${ }^{8}$.

Dispone de catorce consultas médicas (una para cada médico, una de salud a la mujer, una de salud infantil y juvenil, una consulta de apoyo y una consulta polivalente). Incluye en el mismo complejo cinco consultas de enfermería (dos para tratamientos, una de vacunas, una de salud infantil y juvenil y otra para la atención de pacientes diabéticos y anticoagulados). También existen una sala de reuniones, una sala de espera y la secretaría.

El horario de funcionamiento del centro es de 8:00 a 20:00 horas todos los días útiles, con una plantilla de trabajo constituida por diez médicos especialistas de Medicina Familiar y Comunitaria (MFYC), ocho enfermeras y seis administrativos. Este grupo de profesionales de la USF, está dividido a su vez en microequipos de tres elementos, constituidos por médico-enfermera-administrativo. Cada paciente tiene asignada una enfermera que coordina su horario con el de su médico.

La programación del horario de la agenda del médico es libre, siempre y cuando se cumpla el número de 35 horas semanales. La plantilla de la USF se reparte previo acuerdo en horarios de mañana y tarde para garantizar la atención de calidad a los usuarios de la misma, estando cinco médicos de mañana y cinco de tardes. La asistencia de cada médico suele distribuirse aproximadamente en dos o tres jornadas de mañana y dos o tres de tardes, alguna de ella con solapamiento de jornada. En la figura 2 podemos ver un ejemplo de la agenda de un médico de la USF.

\section{3. undonde de saúde famiuar}

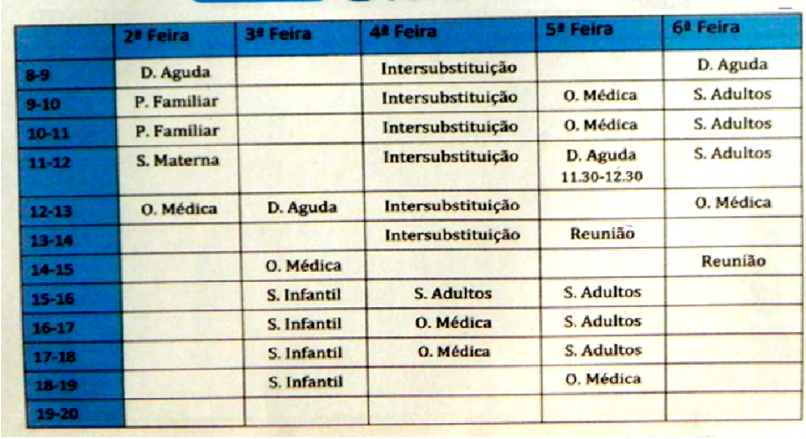

Figura 2. Modelo de agenda de trabajo 
De los médicos, ocho son orientadores de formación en MFYC y aseguran desde enero de 2013 la formación de doce internos. Además, presta formación a otros residentes de Pediatría y especialidades en el año común (año de troncalidad común para las especialidades médicas de los residentes), así como a estudiantes de Medicina y Enfermería.

Respecto a la oferta asistencial, son varios los tipos de consulta que la USF ofrece: consulta de doença aguda, consulta de saúde de adultos, consulta de saúde infantil, consulta de saúde materna, consulta de planeamento familiar, visitas domiciliaras y consultas de intersubstituçao. En todas ellas profundizaremos posteriormente.

Las consultas pueden ser concertadas por el médico, la enfermera o por iniciativa del paciente vía telefónica, por correo electrónico, presencialmente en la secretaría o por el servicio de citas online. Las consultas de doencia aguda y de intersubstituçao son fijadas presencialmente en el propio día. Las primeras tienen un periodo de una hora al día en horario variable según el médico.

A continuación enumeraremos las características de cada uno de los tipos de consulta de la USF:

- Consulta de Saúde de Adultos. Este tipo de consultas se destina a los pacientes con edad superior a 18 años y tienen como principales objetivos la vigilancia y promoción de la salud y el seguimiento de los problemas crónicos. Incluye del mismo modo la prevención y rastreo de enfermedades oncológicas.

- Consulta de Saúde Infantil. Dirigidas a todo paciente con edad inferior o igual a 18 años. Su principal finalidad es el seguimiento de la salud durante la infancia y la adolescencia. En total se realizan 17 consultas desde el nacimiento hasta la adolescencia.

- Consulta de Saúde Materna. Engloba la vigilancia durante el embarazo y la revisión del puerperio. La vigilancia de la gestación puede ser realizada en la USF si es de bajo riesgo prenatal (determinado por el índice de Goodwin modificado) ${ }^{9}$. La periodicidad de las consultas deberá ser mensual hasta las 32 semanas y quincenal entre las 32 y las 36 semanas. La derivación para interconsulta a las consultas de Obstetricia se realiza a las 32-33 semanas para garantizar una primera visita hospitalaria entre las 35 y 36 semanas. El hospital da la posibilidad a todas las embarazadas de un screening bioquímico de cromosopatías del primer trimestre para diagnóstico prenatal. Se explica a la embarazada en qué consiste y la posibilidad de acudir, siendo realizada la derivación a las 12 semanas si la embarazada estuviera de acuerdo. La consulta del puerperio debe ser realizada a las 6 semanas del postparto.

- Consulta de Planeamento Familiar. Acoge a las mujeres en edad fértil (hasta los 54 años) y a los hombres sin límite de edad. Sus objetivos son la promoción de la sexualidad saludable y segura, el control de la fecundidad según el deseo de la pareja, la preparación para la maternidad y la paternidad responsables, así como reducir la morbimortalidad perinatal, materna e infantil y la incidencia de las enfermedades de transmisión sexual y sus consecuencias.

- Doença Aguda. Se trata de consultas no programadas, abiertas diariamente a todos los pacientes del cupo, con un período de una hora diaria en horario variable o constante según el día de la semana o el médico. El número de inscripciones es limitado y el paciente será atendido por su Médico de Familia si se inscribe en dicha franja. Se destina especialmente para la resolución de los problemas agudos o considerados urgentes.

- Intersubstituiçao. Corresponden a las consultas no programadas dirigidas a pacientes que precisan una asistencia con relativa urgencia y que por ausencia física o de disponibilidad de su MF, una vez que no se inscribieron durante el horario correspondiente a las consultas de doença aguda, son atendidos por otro MF de la USF.

- Visitas domiciliarias. Las visitas domiciliarias son un componente importante en los cuidados asistenciales prestados por el MF y una oportunidad para evaluar el contexto familiar y social del paciente, así como los recursos de los que dispone para una determinada situación clínica. Se consideran indicación de visita domiciliaria:

- Seguimiento de patologías incapacitantes tras un alta hospitalaria, pacientes terminales, pacientes con alto grado de incapacidad y pacientes con dificultad de accesibilidad.

- Observación de pacientes con gran sufrimiento emocional: alto riesgo de suicidio, 
abandono de las visitas a consultas con alto riesgo para la salud.

- Evaluación de las condiciones de vivienda y sociales que la situación clínica o fase de la vida de la paciente exija (por ejemplo: puérperas o recién nacidos).

- Contactos indirectos. Se trata de visitas no presenciales en las que el MF da respuesta a solicitudes de sus pacientes dejadas en secretaría, referentes a renovación de medicación crónica, credenciales de transporte, tratamientos prolongados, siempre y cuando tenga un conocimiento previo el médico. Junto a la respuesta al pedido, el médico podrá en las siguientes 48-72 horas enviar la información al paciente. Estas suelen ser realizadas al final de la jornada diaria, durante la jornada de Intersubstituiçao o en huecos puntuales de la agenda (retrasos/ausencias de los pacientes). El tiempo dedicado a las mismas varía según las peticiones que reciba el médico aunque el hecho de contar con una administrativa coordinada en el microequipo facilitaba de manera significativa el trámite de las mismas.

En la labor semanal asistencial, uno de los días de atención de cada médico de familia está dedicado a la atención de la mujer sana y embarazada, y otro día a la edad infantil.

Dentro de la Planificación Familiar, se incluyen herramientas terapéuticas como el DIU y el implante hormonal, los cuales son insertados por el propio médico de familia.

La figura tanto de la matrona como del pediatra en el centro de salud no existe. Por todo ello, podemos recalcar que la atención del médico es a toda la familia.

Una de las particularidades de este Sistema de Salud es su modalidad de pago mixto donde se incluyen las taxas moderadoras. Se trata de una tasa que cada paciente debe pagar para recibir asistencia en la USF. La Consultas de Medicina Geral e familiar llevan consigo una tasa de 2,15€, que en el caso de los pacientes mayores de 65 años es de $1,08 €$. Las Consultas de Planeamento Familiar y Consultas de Saúde Materna están exentas de tasas. En el caso de las Consultas de Saúde Infantil hasta los 13 años también se encuentran exentas de tasas.
Casos especiales son los de los pacientes diabéticos, que tienen dos visitas anuales gratuitas, una cada seis meses, donde el médico realiza un seguimiento de su enfermedad conjuntamente con su enfermera en busca de cribar posibles lesiones de órganos diana con especial atención al pie diabético.

En cuanto a la solicitud de las pruebas complementarias, el médico de cabecera tiene libertad de prescripción y solicitud de las mismas, las cuales también tienen un sistema de cobro mixto, donde el Sistema Sanitario cubre un determinado porcentaje de la misma, con excepción de la resonancia magnética que es la única que no se encuentra financiada.

Otra de las particularidades de las USF es el sistema de pago por incentivo a los profesionales. Existen una serie de indicadores tales como proporción de pacientes hipertensos con presión arterial con cifras menores de 150/90, proporción de mujeres con citología realizada en los últimos 3 años, proporción de pacientes con IMC elevado a los que se les ha realizado una consulta de seguimiento de su peso. Cuando el MF cumple estos incentivos, se le añaden una serie de bonificaciones a su sueldo base.

\section{IMPRESIONES PERSONALES}

Respecto al ambiente en el centro, fuimos muy bien recibidos por parte de todos los estamentos de la USF. El hecho de que se trate de un centro con formación activa de residentes fortaleció nuestra integración. De los doce MF que forman parte de la plantilla, diez de ellos son tutores y tienen al menos un residente. Las sesiones clínicas se realizaban los jueves. Una de ellas fue la experiencia de una residente de la Unidad que había realizado una rotación externa en Canarias coincidiendo, con el Congreso Nacional de la SEMFYC (Sociedad Española de Medicina Familiar y Comunitaria), realizado en el mes de junio. Ello ayudó a poder contar experiencias y explicar nuestro sistema conjuntamente.

Durante la rotación pudimos del mismo modo seguir reforzando nuestra actividad de investigación. 
En el año 2014, la reunión Europea de la Wonca tuvo como punto de encuentro la ciudad de Lisboa. Dentro de nuestra formación como médico interno residente, anualmente y con la ayuda de nuestro tutor, la realización de algún trabajo de investigación es una práctica habitual. En esta ocasión, tuvimos la oportunidad de acudir a dicha reunión anual y presentar los estudio titulados "Influenza vaccination adherence of older people who participated in a workshop" y "Use of Internet by adolescents for topics related to medicine" así como asistir a las diferentes mesas de debate que se celebraron.

Aprovechando la estancia en la USF pudimos recopilar una base de datos de los pacientes diabéticos que acudían a la unidad. Con la misma estamos realizando un trabajo comparativo acerca de las posibles diferencias que existen entre el seguimiento y tratamiento de los pacientes diabéticos en la USF de Oeiras en relación con nuestros pacientes de la consulta de Atención Primaria de Albacete.

Además, y para poder comparar dos sistemas de salud diferentes, también hemos aprovechado para iniciar otro estudio que mide el grado de satisfacción de los pacientes con su médico de familia.

La literatura aporta evidencias sobre la satisfacción de los usuarios con las USF. En un estudio realizado en 2009 en catorce Centros de Saúde y dos USF del Alentejo, la mayoría de los usuarios se mostraron satisfechos en cuanto a la infraestructura, la atención, el acceso a los profesionales y las citas. La satisfacción más baja se relacionó con el espacio lúdico para niños ${ }^{10}$. En 2012, los usuarios de las UCSP y USF del ACES Tâmega I Baixo Tâmega hacían una evaluación favorable de los cuidados de salud por medio del cuestionario Europep. Las dimensiones más satisfactorias eran "la relación y la comunicación" (especialmente el tiempo dedicado por el personal de enfermería), los "recursos humanos" (sobre todo la competencia, cortesía y cariño de los enfermeros) y las "instalaciones". Los niveles más bajos de satisfacción, aunque positivos, eran los relativos a la organización de los servicios, la continuidad y cooperación. El tiempo de espera, en la sala, era entre todos los referidos a la organización de los servicios el peor evaluado ${ }^{11}$.

Mención especial merecen nuestras tutoras en la rotación, ya que se volcaron en nuestra formación durante la estancia. La Doctora Susana Corte-Real, tutora de residentes, y con un currículum que incluía dos rotaciones externas en Madrid y Barcelona durante su formación, lo cual hizo que el problema de la barrera idiomática fuera inexistente, y la Doctora Vanda Proença, con otra rotación externa previamente en La Rioja, se involucraron activamente en acogernos como unos miembros más de la USF.

Por todo ello, nos gustaría recalcar de nuevo lo gratificante que ha sido poder realizar una rotación externa en un Sistema de Salud diferente donde la Medicina Familiar se encuentra reforzada. Conocer un nuevo enfoque en el que se realiza la práctica médica amplía la visión de la profesión, del mismo modo que ayudó a seguir potenciando nuestra formación como residente y a fortalecer nuestro plano investigador.

\section{BIBLIOGRAFÍA}

1. Missão para os Cuidados de Saúde Primários. Reforma dos Cuidados de Saúde Primários. Plano Estratégico 2010 - 2011. Lisboa, 2010.

2. Decreto-Lei n 298/2007, de 22 de Agosto. Diário da República $-1^{\text {a }}$ Série, p. 5587-96.

3. Câmara Municipal de Oeiras. Mapa do Concelho de Oeiras. Disponible en: www.cm-oeiras.pt/ [consultado el 20/11/2014]

4. Instituto Nacional de Estatística (INE). Censos 20: resultados definitivos. XIV recenseamento geral da população; IV recenseamento geral da habitação. Disponible en: https:// www.ine.pt/ [consultado el 23/11/2014]

5. Câmara Municipal de Oeiras. Oeiras, Factos e Números. 2009. Disponible en www.cm-oeiras.pt/ [consultado el 20/11/2014]

6. Instituto Nacional de Estatística. Anuário Estatístico da Região de Lisboa 2009. Lisboa: Instituto Nacional de Estatística, I.P.; 2010.

7. Adminitração Central do Sistema de Saúde. Disponible en: http://www.acss.min-saude.pt/ [consultado el 28/5/2015]

8. Unidade de Saúde Familiar S. Juliao. Disponible en: http:// usf-sjuliao.min-saude.pt/Paginas/default.aspx [consultado el 10/2/2015]

9. Manual de Medicina Geral e Familiar. Parte II - Promoção e protecção da saúde. Avaliação e gestão do risco prénatal. Disponible en: http://csgois.web.interacesso.pt/ 
MGFV001MASTER/textos/23/45_texto.html [consultado el 4/4/2015]

10. Mendes F, Mantovani MF, Gemito ML, Lopes MJ. A satisfação dos utentes com os cuidados de saúde primários. Revista de Enfermagem Referência. 2013;
III (9): 17-25.

11. Machado C. Satisfação de utentes do ACES Tâmega I Baixo Tâmega. Disponible en: http://recipp.ipp. pt/handle/10400.22/1899 [consultado el 4/4/2015] 